PROCEEDINGS OF THE

AMERICAN MATHEMATICAL SOCIETY

Volume 129, Number 8, Pages 2261-2271

S 0002-9939(01)06127-5

Article electronically published on March 20, 2001

\title{
AN ASYMMETRIC PUTNAM-FUGLEDE THEOREM FOR UNBOUNDED OPERATORS
}

\author{
JAN STOCHEL
}

(Communicated by Joseph A. Ball)

Dedicated to Professor F. H. Szafraniec on the occasion of his sixtieth birthday

Abstract. The intertwining relations between cosubnormal and closed hyponormal (resp. cohyponormal and closed subnormal) operators are studied. In particular, an asymmetric Putnam-Fuglede theorem for unbounded operators is proved.

\section{INTRODUCTION}

The Putnam-Fuglede theorem [12] says that if a bounded linear mapping intertwines two normal operators, then it intertwines their adjoints. Unfortunately, the Putnam-Fuglede theorem is no longer true for subnormal operators. However, as noticed by Furuta (cf. [2, 3]), an asymmetric version of it remains true for bounded subnormals; furthermore, subnormals can be replaced by more general bounded operators (cf. [17, 18, 14, 15, 8, 21]). In particular, the following holds true: if a bounded linear mapping intertwines a cohyponormal operator with a hyponormal one (both are bounded), then it intertwines their adjoints. In this paper we prove a decomposition theorem (see Theorem 4.2) for intertwining relations between cosubnormal (resp. cohyponormal) and closed hyponormal (resp. subnormal) unbounded operators (cf. 17, 8 for the bounded case). Contrary to what has been shown before (cf. [11, 7, 10]), here the operators in question have arbitrary domains and their spectra may cover the whole complex plane. We also prove an asymmetric version of the Putnam-Fuglede theorem for couples of unbounded operators whose first component is cosubnormal (resp. cohyponormal), while the other one is closed hyponormal (resp. subnormal).

\section{Preliminaries}

All linear spaces taken into consideration in this paper are assumed to be complex. From now on the symbols $\mathcal{H}$ and $\mathcal{K}$ stand for Hilbert spaces. Given a linear mapping $A: \mathcal{H} \supseteq \mathcal{D}(A) \rightarrow \mathcal{K}$, we denote by $\mathcal{N}(A), \mathcal{R}(A), \mathcal{G}(A)$ and $A^{*}$ the kernel, the range, the graph and the adjoint of $A$, respectively. As usual $\left.A\right|_{\mathcal{E}}$ stands for

Received by the editors November 1, 1999.

2000 Mathematics Subject Classification. Primary 47B20; Secondary 47B15.

Key words and phrases. Normal operator, subnormal operator, hyponormal operator, intertwining relation.

This work was supported by KBN grant \# 2P03A 04110.

(C)2001 American Mathematical Society 
the restriction of $A$ to a linear subspace $\mathcal{E}$ of $\mathcal{D}(A) ; \mathcal{E}$ is said to be a core for $A$ if $\mathcal{G}(A) \subseteq \overline{\mathcal{G}\left(\left.A\right|_{\mathcal{E}}\right)}$. Denote by $\boldsymbol{B}(\mathcal{H}, \mathcal{K})$ the set of all bounded linear mappings from $\mathcal{H}$ into $\mathcal{K}$; for simplicity we write $\boldsymbol{B}(\mathcal{H})$ instead of $\boldsymbol{B}(\mathcal{H}, \mathcal{H})$. Set $|X|=\left(X^{*} X\right)^{1 / 2}$ for $X \in \boldsymbol{B}(\mathcal{H}, \mathcal{K})$.

Given an operator $A$ in $\mathcal{H}$ (read: a linear mapping $A: \mathcal{H} \supseteq \mathcal{D}(A) \rightarrow \mathcal{H}$ ), we denote by $\sigma(A)$ the spectrum of $A$. $A$ is said to be pure if the only closed linear subspace of $\mathcal{H}$ reducing $A$ to a normal operator is $\{0\}$. If $\mathcal{L}$ is a closed linear subspace of $\mathcal{H}$, then $A \uparrow_{\mathcal{L}}$ stands for the operator in $\mathcal{L}$ defined by

$$
\mathcal{D}\left(A \uparrow_{\mathcal{L}}\right)=\{f \in \mathcal{L} \cap \mathcal{D}(A): A f \in \mathcal{L}\} \quad \text { and } \quad A \uparrow_{\mathcal{L}} f=A f, \quad f \in \mathcal{D}\left(A \uparrow_{\mathcal{L}}\right) .
$$

If $\mathcal{L}$ reduces $A$ to the operator $B$, then $B=A \uparrow_{\mathcal{L}}$. It may happen that $A \uparrow_{\mathcal{L}}$ is densely defined in $\mathcal{L}$ though $\mathcal{L}$ is not invariant for $A$, i.e. $A(\mathcal{L} \cap \mathcal{D}(A)) \nsubseteq \mathcal{L}$.

Example 1.1. Let $f_{1}$ be a normalized vector in $\mathcal{H}$. Set $\mathcal{L}=\left\{f_{1}\right\}^{\perp}$. Let $A_{0}$ be an unbounded closed densely defined operator in $\mathcal{L}$. Take a normalized vector $e_{1} \in \mathcal{L} \backslash \mathcal{D}\left(A_{0}\right)$. Define the operator $A$ in $\mathcal{H}$ by: $\mathcal{D}(A)=\mathcal{D}\left(A_{0}\right) \dot{+} \mathbb{C} \cdot e_{1} \dot{+} \mathbb{C} \cdot f_{1}$ and $A\left(h+\lambda e_{1}+\mu f_{1}\right)=A_{0} h+\lambda f_{1}$ for $h \in \mathcal{D}\left(A_{0}\right)$ and $\lambda, \mu \in \mathbb{C}$. It is clear that $A$ is closed and densely defined. Moreover, one can check that $\mathcal{D}\left(A \uparrow_{\mathcal{L}}\right)=\mathcal{D}\left(A_{0}\right) \varsubsetneqq$ $\mathcal{D}\left(A_{0}\right) \dot{+} \mathbb{C} \cdot e_{1}=\mathcal{L} \cap \mathcal{D}(A) \varsubsetneqq \mathcal{D}(A), e_{1} \in \mathcal{L} \cap \mathcal{D}(A)$ and $A e_{1}=f_{1} \notin \mathcal{L}$.

A densely defined operator $A$ in $\mathcal{H}$ is said to be hyponormal if $\mathcal{D}(A) \subseteq \mathcal{D}\left(A^{*}\right)$ and $\left\|A^{*} f\right\| \leq\|A f\|$ for $f \in \mathcal{D}(A)$. We say that a densely defined operator $S$ in $\mathcal{H}$ is subnormal if there is a Hilbert space $\mathcal{L} \supseteq \mathcal{H}$ and a normal operator $N$ in $\mathcal{L}$ such that $S \subseteq N$. It is well-known that each subnormal operator is hyponormal and that each hyponormal operator is closable (see [4, 11, 5, 6] for more details).

We begin our considerations by proving a fact which is well-known for bounded hyponormal operators (cf. [1]; see also [5. Proposition 1] for the reverse result).

Proposition 1.2. If $T$ is a hyponormal operator in $\mathcal{H}$, then there exists a contraction $C \in \boldsymbol{B}(\mathcal{H})$ such that $T \subseteq T^{*} C$.

Proof. Since $\left\|T^{*} f\right\| \leq\|T f\|$ for $f \in \mathcal{D}(T) \subseteq \mathcal{D}\left(T^{*}\right)$, there exists a contraction $D^{\prime} \in \boldsymbol{B}\left(\overline{\mathcal{R}(T)}, \overline{\mathcal{R}\left(T^{*}\right)}\right)$ such that $D^{\prime} T \subseteq T^{*}$. Let $D \in \boldsymbol{B}(\mathcal{H})$ be any contraction which extends $D^{\prime}$ (e.g. set $D f=0$ for $f \in \mathcal{H} \ominus \overline{\mathcal{R}(T)}$ ). Then $D T \subseteq T^{*}$. Taking adjoints in the last inclusion and exploiting the closability of $T$, we get $T \subseteq T^{* *} \subseteq$ $(D T)^{*}=T^{*} D^{*}$. This gives us the conclusion with $C=D^{*}$.

The next result offers a sufficient condition for a hyponormal operator to be reduced by a closed subspace.

Proposition 1.3. Let $\mathcal{L}$ be a closed linear subspace of $\mathcal{H}$ and let $T$ be a closed hyponormal operator in $\mathcal{H}$ such that $\left.T\right|_{\mathcal{L}}$ is densely defined in $\mathcal{L}$. Then:

(i) $T \Gamma_{\mathcal{L}}$ is a closed hyponormal operator in $\mathcal{L}$,

(ii) $\mathcal{L}$ reduces $T$ to $\left.T\right|_{\mathcal{L}}$ provided $T \prod_{\mathcal{L}}$ is normal.

Proof. (i) Since $T$ is closed, it is easily seen that the operator $S:=T \uparrow_{\mathcal{L}}$ is closed as well. We now show that

$$
\left.P T^{*}\right|_{\mathcal{D}(S)} \subseteq S^{*}
$$

where $P$ is the orthogonal projection of $\mathcal{H}$ onto $\mathcal{L}$. Indeed, by the hyponormality of $T$, we have $\mathcal{D}(S) \subseteq \mathcal{D}\left(T^{*}\right)$, so $\langle f, S g\rangle=\left\langle T^{*} f, g\right\rangle=\left\langle P T^{*} f, g\right\rangle$ for $f, g \in \mathcal{D}(S)$. This, in turn, implies (1.1). 
Using (1.1) and the hyponormality of $T$ we get

$$
\left\|S^{*} f\right\|=\left\|P T^{*} f\right\| \leq\left\|T^{*} f\right\| \leq\|T f\|=\|S f\|, \quad f \in \mathcal{D}(S) .
$$

Hence $S$ is hyponormal.

(ii) It follows from the normality of $S$ and (1.2) that $\left\|P T^{*} f\right\|=\left\|T^{*} f\right\|$ for $f \in \mathcal{D}(S)$. Hence $T^{*}(\mathcal{D}(S)) \subseteq \mathcal{L}$ and, by (1.1), we have $S^{*} f=T^{*} f$ for $f \in \mathcal{D}(S)$. Applying the latter equality, we obtain

$$
\left\langle P f, S^{*} g\right\rangle=\left\langle f, S^{*} g\right\rangle=\left\langle f, T^{*} g\right\rangle=\langle T f, g\rangle=\langle P T f, g\rangle, \quad f \in \mathcal{D}(T), g \in \mathcal{D}(S),
$$

which implies $P(\mathcal{D}(T)) \subseteq \mathcal{D}\left(S^{* *}\right)=\mathcal{D}(S) \subseteq \mathcal{D}(T)$ and $T P f=S P f=S^{* *} P f=$ $P T f$ for $f \in \mathcal{D}(T)$. Thus $P T \subseteq T P$, which shows that $\mathcal{L}$ reduces $T$ to $S$.

Part (ii) of Proposition [1.3 is no longer true for arbitrary operators $T$.

Example 1.4. Let $e_{0}$ be a normalized vector in $\mathcal{H}$. Set $\mathcal{L}=\left\{e_{0}\right\}^{\perp}$. Take a normalized vector $f_{0}$ in $\mathcal{L}$. Define $T \in \boldsymbol{B}(\mathcal{H})$ by $T h=h-\left\langle h, e_{0}\right\rangle\left(e_{0}+f_{0}\right)$ for $h \in \mathcal{H}$. One can show that $\|T\|=\sqrt{2}$ (use the fact that $\mathcal{H}=\mathcal{L} \dot{+} \mathbb{C} \cdot\left(e_{0}+f_{0}\right)$ ). Since $T h=h$ for $h \in \mathcal{L}$, we conclude that $T \uparrow_{\mathcal{L}}$ is normal. However $\mathcal{L}$ does not reduce $T$ as $T e_{0}=-f_{0}$.

Our next goal is to characterize linear mappings $T$ satisfying the condition $\mathcal{D}(T) \subseteq \mathcal{R}\left(T^{*}\right)$. Let us begin with

Lemma 1.5. If $T: \mathcal{H} \supseteq \mathcal{D}(T) \rightarrow \mathcal{K}$ is a closed densely defined linear mapping, then $\mathcal{H}=\mathcal{D}(T)+\mathcal{R}\left(T^{*}\right)$.

Proof. It is known that $\mathcal{H} \oplus \mathcal{K}=\mathcal{G}(T) \oplus W \mathcal{G}\left(T^{*}\right)$ (cf. [20. Theorem 4.16]), where $W: \mathcal{K} \oplus \mathcal{H} \rightarrow \mathcal{H} \oplus \mathcal{K}$ is given by $W(k, h)=(-h, k)$ for $h \in \mathcal{H}$ and $k \in \mathcal{K}$. If $f \in \mathcal{H}$, then there exist $h \in \mathcal{D}(T)$ and $k \in \mathcal{D}\left(T^{*}\right)$ such that

$$
(f, 0)=(h, T h) \oplus W\left(k, T^{*} k\right)=\left(h-T^{*} k, T h+k\right),
$$

so $f=h-T^{*} k \in \mathcal{D}(T)+\mathcal{R}\left(T^{*}\right)$.

It is easily seen that Lemma 1.5 is no longer true for operators which are not closed (set $T h=0$ for $h \in \mathcal{D}$, where $\mathcal{D}$ is a dense proper linear subspace of $\mathcal{H}$ ).

Corollary $1.6([9$, Theorem 3.3]). If $T: \mathcal{H} \supseteq \mathcal{D}(T) \rightarrow \mathcal{K}$ is a closed densely defined linear mapping, then $\mathcal{R}(T) \subseteq \mathcal{D}\left(T^{*}\right)$ if and only if $T \in \boldsymbol{B}(\mathcal{H}, \mathcal{K})$.

Proof. If $\mathcal{R}(T) \subseteq \mathcal{D}\left(T^{*}\right)$, then Lemma 1.5 yields $\mathcal{D}\left(T^{*}\right)=\mathcal{H}$, so by the closed graph theorem $T \in \boldsymbol{B}(\mathcal{H}, \mathcal{K})$.

Let $T: \mathcal{H} \supseteq \mathcal{D}(T) \rightarrow \mathcal{K}$ be a linear mapping. Define a new linear mapping $\tilde{T}: \mathcal{H} \supseteq \mathcal{D}(\tilde{T}) \rightarrow \overline{\mathcal{R}(T)}$ by $\mathcal{D}(\tilde{T})=\mathcal{D}(T)$ and $\tilde{T} h=T h$ for $h \in \mathcal{D}(\tilde{T})$. It is clear that $\mathcal{N}(\tilde{T})=\mathcal{N}(T), \mathcal{R}(\tilde{T})=\mathcal{R}(T), \mathcal{G}(\tilde{T})=\mathcal{G}(T)$ and $\tilde{T}=Q_{T} T$, where $Q_{T}: \mathcal{K} \rightarrow \overline{\mathcal{R}(T)}$ is the orthogonal projection of $\mathcal{K}$ onto $\overline{\mathcal{R}(T)}$. If $T$ is densely defined, then obviously $\mathcal{N}\left(\tilde{T}^{*}\right)=\{0\}$.

Theorem 1.7. If $T: \mathcal{H} \supseteq \mathcal{D}(T) \rightarrow \mathcal{K}$ is a closed densely defined linear mapping and $\mathcal{H} \neq\{0\}$, then the following conditions are equivalent:

(i) $\mathcal{D}(T) \subseteq \mathcal{R}\left(T^{*}\right)$,

(ii) $\mathcal{R}\left(T^{*}\right)=\mathcal{H}$, 
(iii) $\mathcal{N}(T) \varsubsetneqq \mathcal{D}(T)$ and $\sup _{f \in \mathcal{D}(T) \backslash \mathcal{N}(T)} \frac{|\langle h, f\rangle|}{\|T f\|}<\infty$ for every $h \in \mathcal{H}$,

(iv) $\tilde{T}^{*-1} \in \boldsymbol{B}(\mathcal{H}, \overline{\mathcal{R}(T)})$,

(v) $\mathcal{N}(T)=\{0\}$ and $\tilde{T}^{-1} \in \boldsymbol{B}(\overline{\mathcal{R}(T)}, \mathcal{H})$.

Proof. There is no loss of generality in assuming that $T=\tilde{T}$ and $\overline{\mathcal{R}(T)}=\mathcal{K}$ because, by [16, Theorem 1], $\mathcal{R}\left(T^{*}\right)=\mathcal{R}\left(\tilde{T}^{*}\right)$.

(i) $\Rightarrow$ (ii). By Lemma 1.5, we have $\mathcal{H}=\mathcal{D}(T)+\mathcal{R}\left(T^{*}\right) \subseteq \mathcal{R}\left(T^{*}\right)$, so $\mathcal{R}\left(T^{*}\right)=\mathcal{H}$.

(ii) $\Leftrightarrow$ (iii). One can show that $T$ satisfies (iii) if and only if for every $h \in \mathcal{H}$ there exists $c_{h} \geq 0$ such that $|\langle h, f\rangle| \leq c_{h}\|T f\|$ for all $f \in \mathcal{D}(T)$. By [16, Theorem 1] this is equivalent to $\mathcal{R}\left(T^{*}\right)=\mathcal{H}$.

(ii) $\Rightarrow$ (iv). Since $T^{*-1}$ is a closed linear mapping with $\mathcal{D}\left(T^{*-1}\right)=\mathcal{H}$, the closed graph theorem yields $T^{*-1} \in \boldsymbol{B}(\mathcal{H}, \mathcal{K})$.

The implications (iv) $\Rightarrow$ (ii) and (ii) $\Rightarrow$ (i) are obvious.

(i) $\Leftrightarrow(\mathrm{v})$. According to (i) $\Rightarrow($ ii $), \mathcal{N}(T)=\{0\}$ whenever $\mathcal{D}(T) \subseteq \mathcal{R}\left(T^{*}\right)$. Thus there is no loss of generality in assuming that $\mathcal{N}(T)=\{0\}$. Then $T^{-1}$ is a closed densely defined linear mapping and $\left(T^{-1}\right)^{*}=T^{*-1}$. Hence $\mathcal{D}\left(\left(T^{-1}\right)^{*}\right)=\mathcal{R}\left(T^{*}\right)$ and $\mathcal{R}\left(T^{-1}\right)=\mathcal{D}(T)$. Applying Corollary 1.6 to $T^{-1}$ we get (i) $\Leftrightarrow(\mathrm{v})$.

Corollary 1.8. Let $T: \mathcal{H} \supseteq \mathcal{D}(T) \rightarrow \mathcal{K}$ be a closed densely defined linear mapping. If $\overline{\mathcal{R}(T)}=\mathcal{K}($ resp. $\mathcal{N}(T)=\{0\})$, then the following conditions are equivalent:

(i) $\mathcal{D}(T) \subseteq \mathcal{R}\left(T^{*}\right)\left(\right.$ resp. $\left.\mathcal{D}\left(T^{*}\right) \subseteq \mathcal{R}(T)\right)$,

(ii) $\mathcal{R}\left(T^{*}\right)=\mathcal{H}($ resp. $\mathcal{R}(T)=\mathcal{K})$,

(iii) $\mathcal{N}(T)=\{0\}$ and $\mathcal{R}(T)=\mathcal{K}$ or equivalently $\overline{\mathcal{R}(T)}=\mathcal{K}$ and $\mathcal{R}\left(T^{*}\right)=\mathcal{H}$,

(iv) $\mathcal{N}(T)=\{0\}$ and $T^{-1} \in \boldsymbol{B}(\mathcal{K}, \mathcal{H})$.

Moreover, if $\mathcal{K}=\mathcal{H}, \mathcal{N}(T)=\{0\}$ and $\overline{\mathcal{R}(T)}=\mathcal{H}$, then $\mathcal{D}(T) \subseteq \mathcal{R}\left(T^{*}\right)$ if and only if $\mathcal{D}\left(T^{*}\right) \subseteq \mathcal{R}(T)$ if and only if $0 \notin \sigma(T)$.

\section{INTERTWINING NORMAL WITH HYPONORMAL OPERATORS}

In this section we investigate intertwining relations between normal and hyponormal operators. We begin with the question of preserving cores by selfadjoint intertwining operators (see [11, Theorem 2.5] for a related result).

Proposition 2.1. Let $\mathcal{E}$ be a core for a closed densely defined operator $A$ in $\mathcal{H}$ and let $X \in \boldsymbol{B}(\mathcal{H})$ be a selfadjoint operator with $\mathcal{N}(X)=\{0\}$. If $X A \subseteq A X$, then $X(\mathcal{E})$ is a core for $A$.

Proof. Take $f \in \mathcal{D}(A)$ such that $f \oplus A f$ is orthogonal in $\mathcal{H} \oplus \mathcal{H}$ to $X g \oplus A X g$ for every $g \in \mathcal{E}$. Then, according to $X=X^{*}$ and $X A \subseteq A X$, we see that

$$
\begin{aligned}
\langle X f, g\rangle=\langle f, X g\rangle & =-\langle A f, A X g\rangle \\
& =-\langle A f, X A g\rangle \\
& =-\langle X A f, A g\rangle \\
& =-\langle A X f, A g\rangle, \quad g \in \mathcal{E} .
\end{aligned}
$$

Since $\mathcal{E}$ is a core for $A$, we conclude that $A X f \in \mathcal{D}\left(A^{*}\right)$ and $A^{*} A X f=-X f$. However $A^{*} A$ is positive and selfadjoint, so $X f=0$. Since $\mathcal{N}(X)=\{0\}$, we get $f=0$. This completes the proof.

The next result can be extracted from [13, Theorem 1] (it is easily seen that [13. Theorem 1] remains true for unbounded normal operators). 
Theorem 2.2. Let $E$ be the spectral measure of a normal operator $N$. If $\sigma$ is a Borel subset of $\mathbb{C}$ and $f \in \bigcap_{z \in \sigma} \mathcal{R}(z-N)$, then $E(\sigma) f=0$.

We are now in a position to prove the crucial result for our further investigations. The idea of its proof is taken from [17, Theorem 1]).

Theorem 2.3. Assume that $N$ is a normal operator in $\mathcal{H}, T$ is a closed hyponormal operator in $\mathcal{K}$ and $X \in \boldsymbol{B}(\mathcal{H}, \mathcal{K})$ is such that $X N \subseteq T X$. Then:

(i) $|X| N \subseteq N|X|$,

(ii) $N=T$ provided $\mathcal{K}=\mathcal{H}, X \geq 0$ and $\mathcal{N}(X)=\{0\}$.

Proof. (i) Let $E$ be the spectral measure of $N$. Take a compact subset $\sigma$ of $\mathbb{C}$ and $f \in \mathcal{H}_{\sigma}:=\mathcal{R}(E(\sigma))$. The space $\mathcal{H}_{\sigma}$ reduces $\lambda-N$ and $\mathcal{H}_{\sigma} \subseteq \mathcal{D}(\lambda-N)$ for $\lambda \in \mathbb{C}$. Define the function $\varphi: \mathbb{C} \backslash \sigma \longrightarrow \mathcal{H}_{\sigma}$ by

$$
\varphi(\lambda)=\int_{\sigma} \frac{1}{\lambda-z} E(d z) f=\left(\lambda-N\left\lceil\mathcal{H}_{\sigma}\right)^{-1} f, \quad \lambda \notin \sigma .\right.
$$

Then

$$
(\lambda-N) \varphi(\lambda)=f, \quad \lambda \notin \sigma .
$$

According to $X N \subseteq T X$, we have

$$
X(\lambda-N) \subseteq(\lambda-T) X, \quad \lambda \in \mathbb{C} .
$$

Combining (2.1) and (2.2), we get $X f=X(\lambda-N) \varphi(\lambda)=(\lambda-T) X \varphi(\lambda)$ for $\lambda \notin \sigma$. Hence

$$
X^{*} X f=X^{*}(\lambda-T) X \varphi(\lambda), \quad \lambda \notin \sigma .
$$

Since $\lambda-T$ is hyponormal, we conclude from Proposition 1.2 that there exists a contraction $C_{\lambda} \in \boldsymbol{B}(\mathcal{K})$ such that

$$
\lambda-T \subseteq(\lambda-T)^{*} C_{\lambda}, \quad \lambda \in \mathbb{C} .
$$

Taking adjoints in (2.2), we get

$$
X^{*}(\lambda-T)^{*} \subseteq(\lambda-N)^{*} X^{*}, \quad \lambda \in \mathbb{C} .
$$

It follows from (2.3), 2.4) and (2.5) that

$$
\begin{aligned}
X^{*} X f=X^{*}(\lambda-T) X \varphi(\lambda) & =X^{*}(\lambda-T)^{*} C_{\lambda} X \varphi(\lambda) \\
& =(\lambda-N)^{*} X^{*} C_{\lambda} X \varphi(\lambda), \quad \lambda \notin \sigma .
\end{aligned}
$$

Hence $X^{*} X f$ belongs to $\bigcap_{z \in \mathbb{C} \backslash \sigma^{*}} \mathcal{R}\left(z-N^{*}\right)$, where $\sigma^{*}=\{z: \bar{z} \in \sigma\}$. According to Theorem $\left[2.2\right.$, we have $E(\mathbb{C} \backslash \sigma) X^{*} X f=0$ (because the spectral measure $F$ of $N^{*}$ is related to $E$ via $F(\tau)=E\left(\tau^{*}\right)$ for every Borel subset $\tau$ of $\mathbb{C}$ ) or equivalently $X^{*} X f=E(\sigma) X^{*} X f$. Since $f$ was an arbitrary element of $\mathcal{H}_{\sigma}$, we conclude that $X^{*} X\left(\mathcal{H}_{\sigma}\right) \subseteq \mathcal{H}_{\sigma}$. However $X^{*} X \geq 0$, so $X^{*} X E(\sigma)=E(\sigma) X^{*} X$. This, in turn, implies that $|X| E(\sigma)=E(\sigma)|X|$ for every compact subset $\sigma$ of $\mathbb{C}$. Since $E$ is a regular measure, the latter equality holds for every Borel subset $\sigma$ of $\mathbb{C}$, which implies (i).

(ii) Since $X \geq 0$, we conclude from (i) that $N X f=X N f=T X f$ for $f \in \mathcal{D}(N)$. Thus

$$
\left.N\right|_{X \mathcal{D}(N)} \subseteq T
$$


However, according to Proposition 2.1 $X \mathcal{D}(N)$ is a core for $N$, so taking closures in (2.6) leads to $N \subseteq T$. Hence $T^{*} \subseteq N^{*}$ and, by the hyponormality of $T$, we have $\mathcal{D}(T) \subseteq \mathcal{D}\left(T^{*}\right) \subseteq \mathcal{D}\left(N^{*}\right)=\mathcal{D}(N)$. This gives us $N=T$.

\section{More on INTERTWINING RELATIONS}

In this section we continue the study of intertwining relations. Let us first collect notations and definitions which play the basic role in the rest of the paper.

Given $X \in \boldsymbol{B}(\mathcal{H}, \mathcal{K})$, we set $\mathcal{I}(X):=\overline{\mathcal{R}\left(X^{*}\right)}=\overline{\mathcal{R}(|X|)}$ and $\mathcal{F}(X):=\overline{\mathcal{R}(X)}$. Let $X=\mathfrak{i}_{X}|X|$ be the polar decomposition of $X$, i.e. $\mathfrak{i}_{X} \in \boldsymbol{B}(\mathcal{H}, \mathcal{K})$ is the partial isometry with $\mathcal{N}\left(\mathfrak{i}_{X}\right)=\mathcal{N}(X)$, which maps isometrically $\mathcal{I}(X)$ onto $\mathcal{F}(X)$. Denote by $\mathfrak{u}_{X}$ and $\widehat{X}$ the bounded linear mappings from $\mathcal{I}(X)$ into $\mathcal{F}(X)$ defined by $\mathfrak{u}_{X}:=\left.\mathfrak{i}_{X}\right|_{\mathcal{I}(X)}$ and $\widehat{X}:=\left.X\right|_{\mathcal{I}(X)}$. Then $\mathfrak{u}_{X}$ is a unique unitary isomorphism in $\boldsymbol{B}(\mathcal{I}(X), \mathcal{F}(X))$ such that

$$
\mathfrak{u}_{X}|X| f=X f, \quad f \in \mathcal{H}
$$

Lemma 3.1. If $X \in \boldsymbol{B}(\mathcal{H}, \mathcal{K})$, then

(i) $\mathcal{N}(\widehat{X})=\{0\}$

(ii) $\mathcal{R}(X)=\mathcal{R}(\widehat{X})$,

(iii) $\widehat{X}^{*}=\widehat{X}^{*}$,

(iv) $\widehat{|X|}=|\widehat{X}|$,

(v) $\mathfrak{i}_{\widehat{X}}=\mathfrak{u}_{\widehat{X}}=\mathfrak{u}_{X}$.

Proof. The proof of (i), (ii) and (iii) is left to the reader.

(iv) Since $\mathcal{I}(X)=\mathcal{I}(|X|)$ reduces $|X|$ and $\widehat{X^{*}}=\widehat{X}^{*}$, we get $|\widehat{X}|=\left(\widehat{X^{*}} \widehat{X}\right)^{1 / 2}=$ $\left(\left.\left(X^{*} X\right)\right|_{\mathcal{I}(X)}\right)^{1 / 2}=\mid X \|_{\mathcal{I}(X)}=\widehat{|X|}$, which proves (iv).

(v) It follows from (i) and (ii) that

$$
\mathcal{I}(X)=\mathcal{I}(\widehat{X}) \quad \text { and } \quad \mathcal{F}(X)=\mathcal{F}(\widehat{X}) .
$$

On the other hand, by (iv) and (3.1), we have $\mathfrak{u}_{X}|\widehat{X}| f=\mathfrak{u}_{X} \widehat{|X|} f=\mathfrak{u}_{X}|X| f=X f=$ $\widehat{X} f$ for $f \in \mathcal{I}(X)$. This implies that $\mathfrak{u}_{\widehat{X}}=\mathfrak{u}_{X}$ due to the uniqueness of $\mathfrak{u}_{\widehat{X}}$. The equality $\mathfrak{i}_{\widehat{X}}=\mathfrak{u}_{\widehat{X}}$ is a consequence of (3.2).

If $A$ and $B$ are operators in $\mathcal{H}$ and $\mathcal{K}$, respectively, and $X \in \boldsymbol{B}(\mathcal{H}, \mathcal{K})$, then we set $A_{X}:=A \uparrow_{\mathcal{I}(X)}$ and $B^{X}:=B \uparrow_{\mathcal{F}(X)}$. To shorten notation, we write $A_{X}^{*}$ instead of $\left(A_{X}\right)^{*}$. In case $X$ intertwines $A^{*}$ and $B, A_{X}$ and $B^{X}$ have the following properties.

Theorem 3.2. Let $A$ and $B$ be closed densely defined operators in $\mathcal{H}$ and $\mathcal{K}$, respectively, and let $X \in \boldsymbol{B}(\mathcal{H}, \mathcal{K})$ be such that $X A^{*} \subseteq B X$.

(i) If $\mathcal{I}(X)$ reduces $A$, then $B^{X}$ is closed densely defined in $\mathcal{F}(X)$ and

$$
\widehat{X} A_{X}^{*} \subseteq B^{x} \widehat{X} .
$$

(ii) If $\mathcal{I}(X)$ and $\mathcal{F}(X)$ reduce $A$ and $B$ to normal operators, respectively, then

$$
\begin{gathered}
X A \subseteq B^{*} X, \quad|X| A \subseteq A|X|, \quad\left|X^{*}\right| B \subseteq B\left|X^{*}\right|, \\
A_{X}^{*}=\mathfrak{u}_{X}^{*} B^{X} \mathfrak{u}_{X} .
\end{gathered}
$$

Proof. (i) The closedness of $B^{x}$ follows directly from that of $B$. We now show that

$$
\widehat{X}\left(\mathcal{D}\left(A_{X}^{*}\right)\right) \subseteq X\left(\mathcal{D}\left(A^{*}\right)\right) \subseteq \mathcal{D}\left(B^{X}\right)
$$


Indeed, since $A_{X}^{*}=\left.A^{*}\right|_{\mathcal{I}(X)}$, we get $\widehat{X}\left(\mathcal{D}\left(A_{X}^{*}\right)\right)=X\left(\mathcal{D}\left(A^{*}\right) \cap \mathcal{I}(X)\right) \subseteq X\left(\mathcal{D}\left(A^{*}\right)\right)$. According to $X A^{*} \subseteq B X$, we have $X\left(\mathcal{D}\left(A^{*}\right)\right) \subseteq \mathcal{D}(B) \cap \mathcal{F}(X)$ and $B X f=X A^{*} f \in$ $\mathcal{F}(X)$ for $f \in \mathcal{D}\left(A^{*}\right)$. Hence $X\left(\mathcal{D}\left(A^{*}\right)\right) \subseteq \mathcal{D}\left(B^{X}\right)$, which proves (3.5).

Since the operator $A^{*}$ is densely defined in $\mathcal{H}$, we conclude from (B.5) that $\mathcal{F}(X)=\overline{X\left(\overline{\left.\mathcal{D}\left(A^{*}\right)\right)}\right.}=\overline{X\left(\mathcal{D}\left(A^{*}\right)\right)} \subseteq \overline{\mathcal{D}\left(B^{X}\right)}$, which shows that $B^{X}$ is densely defined in $\mathcal{F}(X)$. Applying once more the equality $A_{X}^{*}=\left.A^{*}\right|_{\mathcal{I}(X)}$ and (3.5), we obtain $\widehat{X} A_{X}^{*} f=X A^{*} f=B X f=B^{x} \widehat{X} f$ for $f \in \mathcal{D}\left(A_{X}^{*}\right)$. This completes the proof of (i).

(ii) Applying the Putnam-Fuglede theorem (cf. [12, Lemma]) to (3.3), we obtain

$$
\widehat{X} A_{X} \subseteq\left(B^{X}\right)^{*} \widehat{X} .
$$

Therefore, by Lemma $\left[3.1(\mathrm{v})\right.$, we have $\mathfrak{u}_{X}|\widehat{X}| A_{X} \subseteq\left(B^{X}\right)^{*} \mathfrak{u}_{X}|\widehat{X}|$ or, equivalently,

$$
|\widehat{X}| A_{X} \subseteq\left(\mathfrak{u}_{X}^{*}\left(B^{X}\right)^{*} \mathfrak{u}_{X}\right)|\widehat{X}| .
$$

Since, according to Lemma $\widehat{3.1}(\mathrm{i}),($ iv), $\mathcal{N}(|\widehat{X}|)=\mathcal{N}(\widehat{|X|})=\{0\}$, we conclude from (3.7) via Theorem 2.3 that $A_{X}=\mathfrak{u}_{X}^{*}\left(B^{X}\right)^{*} \mathfrak{u}_{X}$ (which implies (3.4) $)$ and

$$
|\widehat{X}| A_{X} \subseteq A_{X}|\widehat{X}| \text {. }
$$

As $X=\widehat{X} \oplus 0, A=A_{X} \oplus A^{\prime}$ and $B=B^{X} \oplus B^{\prime}$ with $A^{\prime}=A \uparrow_{\mathcal{N}(X)}$ and $B^{\prime}=B \uparrow_{\mathcal{N}\left(X^{*}\right)}$, we deduce from (3.6) that

$$
X A \subseteq \widehat{X} A_{X} \oplus 0 \subseteq\left(B^{X}\right)^{*} \widehat{X} \oplus 0=B^{*} X .
$$

It follows from Lemma 3.1 (iv) that $|X|=|\widehat{X}| \oplus 0$. Hence (3.8) leads to

$$
|X| A \subseteq|\widehat{X}| A_{X} \oplus 0 \subseteq A_{X}|\widehat{X}| \oplus 0=A|X| .
$$

Notice that $X A^{*} \subseteq B X$ implies $X^{*} B^{*} \subseteq A X^{*}$. Since $\mathcal{I}\left(X^{*}\right)$ and $\mathcal{F}\left(X^{*}\right)$ reduce $B$ and $A$ to normal operators, respectively, we can replace the linear mappings $X$ and $A$ in (3.9) by $X^{*}$ and $B$, respectively, to get $\left|X^{*}\right| B \subseteq B\left|X^{*}\right|$. This completes the proof.

\section{INTERTWINING COHYPONORMAL WITH HYPONORMAL OPERATORS}

In this section we describe the structure of intertwining relations between cosubnormal and hyponormal (as well as between cohyponormal and subnormal) operators. In particular, we obtain an asymmetric Putnam-Fuglede theorem for unbounded operators. First we consider a special case of intertwining relations.

Proposition 4.1. Assume that $N$ is a normal operator in $\mathcal{H}, T$ is a closed hyponormal operator in $\mathcal{K}$ and $X \in \boldsymbol{B}(\mathcal{H}, \mathcal{K})$ is such that $X N \subseteq T X$. Then $\mathcal{I}(X)$ and $\mathcal{F}(X)$ reduce $N$ and $T$ to normal operators, respectively.

Proof. It follows from Theorem[2.3(i) that $|X| E(\sigma)=E(\sigma)|X|$ for every Borel subset $\sigma$ of $\mathbb{C}, E$ being the spectral measure of $N$. Since $\mathcal{I}(X)=\overline{\mathcal{R}(|X|)}$, we conclude that $\mathcal{I}(X)$ reduces $E$ and, consequently, $N$ as well. According to Theorem 3.2(i), the operator $T^{X}$ is densely defined in $\mathcal{F}(X)$ and

$$
\widehat{X} N_{X} \subseteq T^{x} \widehat{X} .
$$

By Proposition 1.3(i), $T^{x}$ is a closed hyponormal operator in $\mathcal{F}(X)$. Applying Lemma 3.1(v) to (4.1), we get $|\widehat{X}| N_{X} \subseteq\left(\mathfrak{u}_{X}^{*} T^{x} \mathfrak{u}_{X}\right)|\widehat{X}|$. Since $\mathcal{N}(|\widehat{X}|)=\{0\}$ and $\mathfrak{u}_{X}^{*} T^{x} \mathfrak{u}_{X}$ is a closed hyponormal operator, Theorem[2.3](ii) yields $N_{X}=\mathfrak{u}_{X}^{*} T^{x} \mathfrak{u}_{X}$. Hence $T^{X}$, being unitarily equivalent to the normal operator $N_{X}$, is normal as well. 
By Proposition 1.3 (ii), $\mathcal{F}(X)$ reduces $T$ to a normal operator. This completes the proof.

We are now in a position to prove the main result of the paper.

Theorem 4.2. Assume that $A$ is a closed subnormal (resp. a closed hyponormal) operator in $\mathcal{H}, B$ is a closed hyponormal (resp. a closed subnormal) operator in $\mathcal{K}$ and $X \in \boldsymbol{B}(\mathcal{H}, \mathcal{K})$ is such that $X A^{*} \subseteq B X$. Then:

(i) $X A \subseteq B^{*} X$,

(ii) $|X| A \subseteq A|X|$,

(iii) $\left|X^{*}\right| B \subseteq B\left|X^{*}\right|$,

(iv) $\mathcal{I}(X)$ reduces $A$ to the normal operator $A_{X}$,

(v) $\mathcal{F}(X)$ reduces $B$ to the normal operator $B^{X}$,

(vi) $A_{X}^{*}=\mathfrak{u}_{X}^{*} B^{X} \mathfrak{u}_{X}$.

Proof. Case 1. $A$ is subnormal and $B$ is hyponormal.

Let $N$ be a normal extension of $A$ acting in a Hilbert space $\mathcal{L} \supseteq \mathcal{H}$. Define $Y \in \boldsymbol{B}(\mathcal{K}, \mathcal{L})$ by $Y f=X^{*} f$ for $f \in \mathcal{K}$. We show that

$$
\mathcal{F}(X)=\mathcal{F}\left(Y^{*}\right) \text {. }
$$

Indeed, since $Y=J X^{*}$, where $J \in \boldsymbol{B}(\mathcal{H}, \mathcal{L})$ is defined by $J h=h$ for $h \in \mathcal{H}$, we get $Y^{*}=X P$, where $P \in \boldsymbol{B}(\mathcal{L}, \mathcal{H})$ is the orthogonal projection of $\mathcal{L}$ onto $\mathcal{H}$. The latter equality implies (4.2).

(v) Taking adjoints in $X A^{*} \subseteq B X$, we obtain

$$
X^{*} B^{*} \subseteq A X^{*},
$$

which implies $Y B^{*} \subseteq N Y$. This, in turn, forces $Y^{*} N^{*} \subseteq B Y^{*}$. Hence, by (4.2) and Proposition 4.1 we come to the conclusion that $\mathcal{F}(X)=\mathcal{F}\left(Y^{*}\right)$ reduces $B$ to the normal operator $B^{x}$.

(iv) Applying Theorem 3.2(i) to (4.3) and Lemma 3.1(iii), we obtain that $A_{X}=$ $A^{\left(X^{*}\right)}$ is a closed densely defined operator in $\mathcal{I}(X)$ and

$$
\widehat{X}^{*}\left(B^{X}\right)^{*} \subseteq A_{X} \widehat{X}^{*} .
$$

Since $A_{X} \subseteq A \subseteq N$, we see that $A_{X}$ is subnormal and, in consequence, hyponormal. It follows from Proposition 4.1 applied to (4.4) that $\mathcal{I}(X)=\mathcal{F}\left(\widehat{X}^{*}\right)$ reduces $A_{X}$ to a normal operator. Hence $A_{X}$ is normal itself. By Proposition 1.3(ii), $\mathcal{I}(X)$ reduces $A$ to the normal operator $A_{X}$.

The rest of the conclusion follows directly from Theorem [3.2(ii) applied to the intertwining relation $X A^{*} \subseteq B X$.

Case 2. $A$ is hyponormal and $B$ is subnormal.

It follows from $X A^{*} \subseteq B X$ that $X^{*} B^{*} \subseteq A X^{*}$. According to Case $1, \mathcal{I}(X)=$ $\mathcal{F}\left(X^{*}\right)$ reduces $A$ to the normal operator $A_{X}$ and $\mathcal{F}(X)=\mathcal{I}\left(X^{*}\right)$ reduces $B$ to the normal operator $B^{X}$. The rest of the conclusion follows once more from Theorem 3.2(ii) applied to the relation $X A^{*} \subseteq B X$.

\section{Concluding REMARKS AND COMMENTS}

We begin with a result which can be inferred from Theorem 4.2 via the implications:

a) $\mathcal{N}(X)=\{0\} \Rightarrow \mathcal{I}(X)=\mathcal{H}$

b) $\mathcal{N}\left(X^{*}\right)=\{0\} \Rightarrow \mathcal{F}(X)=\mathcal{K}$, 
c) $(\mathcal{K}=\mathcal{H} \wedge X \geq 0 \wedge \mathcal{N}(X)=\{0\}) \Rightarrow \mathfrak{u}_{X}$ is the identity operator on $\mathcal{H}$,

d) $X \neq 0 \Rightarrow(\mathcal{I}(X) \neq\{0\} \wedge \mathcal{F}(X) \neq\{0\})$.

Corollary 5.1. Assume that $A$ is a closed subnormal (resp. a closed hyponormal) operator in $\mathcal{H}, B$ is a closed hyponormal (resp. a closed subnormal) operator in $\mathcal{K}$ and $X \in \boldsymbol{B}(\mathcal{H}, \mathcal{K})$ is such that $X A^{*} \subseteq B X$. Then:

(i) $A$ is normal provided $\mathcal{N}(X)=\{0\}$,

(ii) $B$ is normal provided $\mathcal{N}\left(X^{*}\right)=\{0\}$,

(iii) $B$ and $A^{*}$ are normal and unitarily equivalent provided $\mathcal{N}(X)=\{0\}$ and $\mathcal{N}\left(X^{*}\right)=\{0\}$

(iv) $B=A^{*}$ and $A$ is normal provided $\mathcal{K}=\mathcal{H}, X \geq 0$ and $\mathcal{N}(X)=\{0\}$,

(v) neither $A$ nor $B$ is pure provided $X \neq 0$.

Let us make some comments concerning Corollary 5.1 part (ii) of it extends [7] Theorem 3] (the assumption $\sigma(T) \neq \mathbb{C}$ can be dropped); part (iii) generalizes [11. Theorem 4.1], [11, Proposition 4.2], [11, Corollaries 4.4 and 4.6], [7, Corollary 4] (the assumption $\sigma(N) \neq \mathbb{C}$ can be dropped), 7, Corollary 9] (the assumption $\mathcal{D}\left(S_{j}\right)=\mathcal{D}\left(S_{j}^{*}\right), j=1,2$, can be dropped) and [10 Theorem 3.3] (the operator $C$ does coincides with $\bar{A}$ ); part (iv) generalizes [11, Theorem 4.5], 7, Theorem 8] (the assumptions $\mathcal{D}\left(S_{j}\right)=\mathcal{D}\left(S_{j}^{*}\right), j=1,2$, and $\sigma\left(S_{1}\right) \neq \mathbb{C}$ can be dropped) and 10, Proposition 3.2] (the operator $B$ does coincides with $\bar{A}$ ); finally, part (v) has been applied in [19, Lemma 8.5] to prove that the strong symmetric commutant of a closed hyponormal operator is equal to the orthogonal sum of strong symmetric commutants of its normal and pure parts.

In particular part (iii) of Corollary 5.1 implies that a closed hyponormal operator $A$ which is quasi-similar to a selfadjoint operator $B$ is selfadjoint itself and unitarily equivalent to $B$ (see 11 for the definition of quasi-similarity and Corollary 4.6 therein for a related result).

The last result of the paper is related to Remark 2.6 following [11 Theorem 2.5]. We show that the assumption imposed on the operator $B$ in [11, Remark 2.6] is not weaker than that in [11, Theorem 2.5].

Theorem 5.2. Let $A$ and $B$ be closed densely defined operators in $\mathcal{H}$ and $\mathcal{K}$, respectively, and let $X \in \boldsymbol{B}(\mathcal{H}, \mathcal{K})$ be such that $X A \subseteq B X$. Assume that $\overline{\mathcal{R}(A)}=\mathcal{H}$ and $\overline{\mathcal{R}(X)}=\mathcal{K}$. Then:

(i) $\overline{\mathcal{R}(B)}=\mathcal{K}$,

(ii) $\mathcal{D}(B) \subseteq \mathcal{R}\left(B^{*}\right)$ if and only if $0 \notin \sigma(B)$,

(iii) $0 \notin \sigma(A)$ provided $0 \notin \sigma(B)$ and $X^{-1} \in \boldsymbol{B}(\mathcal{K}, \mathcal{H})$.

Proof. Since $\overline{\mathcal{R}(X)}=\mathcal{K}$ and $\overline{\mathcal{R}(A)}=\mathcal{H}$, we conclude that $\overline{\mathcal{R}(X A)}=\mathcal{K}$. This and $X A \subseteq B X$ lead to $\overline{\mathcal{R}(B)}=\mathcal{K}$. Hence (ii) follows from Corollary 1.8.

Assume that $0 \notin \sigma(B)$ and $X^{-1} \in \boldsymbol{B}(\mathcal{K}, \mathcal{H})$. By $X A \subseteq B X$, we have $A f=$ $X^{-1} X A f=X^{-1} B X f$ for $f \in \mathcal{D}(A)$, so

$$
\begin{aligned}
\|A f\|=\left\|X^{-1} B X f\right\| & \geq\|X\|^{-1}\|B X f\| \\
& \geq\left(\|X\|\left\|B^{-1}\right\|\right)^{-1}\|X f\| \\
& \geq\left(\|X\|\left\|B^{-1}\right\|\left\|X^{-1}\right\|\right)^{-1}\|f\|, \quad f \in \mathcal{D}(A) .
\end{aligned}
$$

This means that $A$ is bounded from below and $\overline{\mathcal{R}(A)}=\mathcal{H}$. Therefore $A^{-1} \in \boldsymbol{B}(\mathcal{H})$, which completes the proof of (iii). 
We close the paper with an example which shows that the assumption $X^{-1} \in$ $\boldsymbol{B}(\mathcal{K}, \mathcal{H})$ could not be removed from part (iii) of Theorem 5.2

Example 5.3. Let $\mathcal{H}$ be a separable Hilbert space with an orthonormal basis $\left\{e_{n}\right\}_{n \in \mathbb{Z}}$ and let $\mathcal{E}$ be the linear span of $\left\{e_{n}\right\}_{n \in \mathbb{Z}}(\mathbb{Z}=\{\ldots,-1,0,1, \ldots\})$. Define the sequence $\boldsymbol{\lambda}=\left\{\lambda_{n}\right\}_{n \in \mathbb{Z}}$ by

$$
\lambda_{n}= \begin{cases}1, & n \leq 0, \\ 1 / n, & n \in 2 \cdot \mathbb{Z}, \quad n \geq 1, \\ 1 / n^{2}, & n \in 2 \cdot \mathbb{Z}+1, \quad n \geq 1 .\end{cases}
$$

Let $B$ and $D_{\boldsymbol{\lambda}}$ be the members of $\boldsymbol{B}(\mathcal{H})$ determined by the conditions $B e_{n}=e_{n+1}$ and $D_{\boldsymbol{\lambda}} e_{n}=\lambda_{n} e_{n}(n \in \mathbb{Z})$, and let $X:=B^{2} D_{\boldsymbol{\lambda}}(\in \boldsymbol{B}(\mathcal{H}))$. Then $\mathcal{N}(B)=\{0\}$, $B^{-1} \in \boldsymbol{B}(\mathcal{H})$ and $\mathcal{N}(X)=\{0\}$ because $\mathcal{N}\left(D_{\boldsymbol{\lambda}}\right)=\{0\}$. Since $X e_{n}=\lambda_{n} e_{n+2}$ for $n \in \mathbb{Z}$, we get $\mathcal{E} \subseteq \mathcal{R}(X)$ and consequently $\overline{\mathcal{R}(X)}=\mathcal{H}$. Set $A=B D_{\mu}$, where $D_{\mu}$ is a unique normal operator in $\mathcal{H}$ determined by the condition $D_{\mu} e_{n}=\mu_{n} e_{n}$ with $\mu_{n}=\lambda_{n} / \lambda_{n+1}(n \in \mathbb{Z})$. The operator $A$ is closed densely defined and unbounded; moreover, $\mathcal{N}(A)=\{0\}$. Since $A e_{n}=\mu_{n} e_{n+1}$ for $n \in \mathbb{Z}$, we get $\mathcal{E} \subseteq \mathcal{R}(A)$ and $A^{-1} e_{n}=\mu_{n-1}^{-1} e_{n-1}$ for $n \in \mathbb{Z}$. Therefore $\overline{\mathcal{R}(A)}=\mathcal{H}$ and the operator $A^{-1}$ is unbounded (because the sequence $\left\{\mu_{n}^{-1}\right\}_{n \in \mathbb{Z}}$ is unbounded). One can check that $X A e_{n}=B X e_{n}$ for $n \in \mathbb{Z}$, hence that $X\left(\left.A\right|_{\mathcal{E}}\right) \subseteq B X$ and finally that $X A \subseteq B X$ (because $\mathcal{E}$ is a core for $A$ and $B$ is closed). Clearly, the operator $X^{-1}$ is unbounded.

\section{REFERENCES}

[1] K. Clancey, Seminormal operators, Lecture Notes in Math., vol. 742, Springer-Verlag, Berlin, Heidelberg, and New York, 1979. MR 81c:47002

[2] T. Furuta, Relaxation of normality in the Fuglede-Putnam theorem, Proc. Amer. Math. Soc. 77 (1979), 324-328. MR 80i:47037

[3] T. Furuta, An extension of the Fuglede-Putnam theorem to subnormal operators using a Hilbert-Schmidt norm inequality, Proc. Amer. Math. Soc. 81 (1981), 240-242. MR 82e:47035

[4] J. Janas, On unbounded hyponormal operators, Ark. Mat. 27 (1989), 273-281. MR 91i:47031

[5] J. Janas, On unbounded hyponormal operators. II, Integr. Equat. Oper. Th. 15 (1992), 470478. MR 93b:47047

[6] J. Janas, On unbounded hyponormal operators. III, Studia Math. 112 (1994), 75-82. MR 95m:47037

[7] K. H. Jin, On unbounded subnormal operators, Bull. Korean Math. Soc. 30 (1993), 65-70. MR 94h: 47041

[8] R.L. Moore, D.D. Rogers and T.T. Trent, A note on intertwining M-hyponormal operators, Proc. Amer. Math. Soc. 83 (1981), 514-516. MR 82j:47033

[9] S. Ôta, Closed linear operators with domain containing their range, Proc. Edinburgh Math. Soc. 27 (1984), 229-233. MR 86e:47002

[10] S. Ôta , A quasi-affine transform of an unbounded operator, Studia Math. 112 (1995), 279284. MR 96f: 47043

[11] S. Ôta and K. Schmüdgen, On some classes of unbounded operators, Integr. Equat. Oper. Th. 12 (1989), 211-226. MR 90d:47027

[12] C. R. Putnam, On normal operators in Hilbert space, Amer. J. Math. 73 (1951), 357-362. MR 12:717f

[13] C. R. Putnam, Ranges of normal and subnormal operators, Michigan Math. J. 18 (1971), 33-36.

[14] M. Radjabalipour, Ranges of hyponormal operators, Illinois J. Math. 21 (1977), 70-75. MR 56:6449

[15] M. Radjabalipour, On majorization and normality of operators, Proc. Amer. Math. Soc. 62 (1977), 105-110. MR 55:3856

[16] Z. Sebestyén, On ranges of adjoint operators in Hilbert space, Acta Sci. Math. (Szeged) 46 (1983), 295-298. MR 85i:47003a 
[17] J. G. Stamplli and B. L. Wadhwa, An asymmetric Putnam-Fuglede theorem for dominant operators, Indiana Univ. Math. J. 25 (1976), 359-365. MR 53:14197

[18] J. G. Stampfli and B. L. Wadhwa, On dominant operators, Mh. Math. 84 (1977), 143-153. MR 56:16428

[19] J. Stochel, Lifting strong commutants of unbounded subnormal operators, preprint 1999.

[20] J. Weidmann, Linear Operators in Hilbert Spaces, Springer-Verlag, Berlin-Heidelberg-New York, 1980. MR 81e:47001

[21] T. Yoshino, Remark on the generalized Putnam-Fuglede theorem, Proc. Amer. Math. Soc. 95 (1985), 571-572. MR 87i:47034

Instytut Matematyki, Uniwersytet Jagielloński, Kraków, Poland

E-mail address: stochel@im.uj.edu.pl 